Berlin and one in Potsdam and with two research institutions, the Konrad-Zuse-Center and the Weierstrass Institute for Applied Analysis and Stochastics. Many Berlin mathematicians are eager to make the congress a success. Much work was already done by the Berlin members of Provisional Organizing Committee whom I thank very much, in particular Professor Grötschel, Vice-President of the Konrad-ZuseCenter and the President of the German Mathematical Society. By his efforts it was possible to preregister for ICM 1998 here in Zuirich using the world wide web. I hope many of you have done so.
It is a great pleasure and honor for me to invite on behalf of the German Mathematical Society the International Congress of Mathematicians to Berlin for the period August 18 to 28,1998 . The united city has many theatres and museums. It has forests and lakes. There is much to do. The average maximum daily temperature in Berlin in August is 23 degrees. Specialists in probability have told me however that in August 1998 Berlin may even be hotter than Zürich 1994. But never mind, I hope to see you all in Berlin in any weather.

\title{
Generalversammlung der IMU
}

\section{von Klaus D. Bierstedt}

Vor jedem "International Congress of Mathematicians (ICM)" findet die Generalversammlung (GA) der Internationalen Mathematischen Union (IMU) statt. So traf man sich in Oakland vor dem ICM 86 (Berkeley) und in Kobe vor dem ICM 90 (Kyoto). Dieses Jahr tagten die Mitglieder des Exekutivkomitees (EC) der IMU und knapp 150 Delegierte aus 60 Lăndern am 31. Juli und 1. August in Luzern. Das EC für die Wahlperiode 199194 besteht aus: J.L. Lions (Präsident), Coates, Mumford (Vizepräsidenten), Palis (Sekretär), Arthur, Dold, Komatsu, Lovasz, Zehnder sowie Fadeev (Ex-Präsident). Der deutschen Delegation (stets vier Vertreter der $D M V$ und einer deutschen Sektion der GAMM) gehörten in Luzern die Herren Aigner, Bierstedt, Grötschel, Hirzebruch und Winkler an.

Vielleicht-aus deutscher Sicht aber bestimmtder wichtigste Tagungsordnungspunkt war die Entscheidung um die Vergabe des ICM 98. Denn der damalige Vorsitzende der Deutschen MathematikerVereinigung, Professor Hirzebruch, hatte 1990 anläBlich der Jahrestagung zum 100-jährigen Bestehen der DMV in Bremen verkündet, daß man sich für Berlin bewerben würde. Bei Bewerbungsschluß lagen dann Angebote nach Berlin und Jerusalem vor.(Eine Bewerbung Pekings traf verspätet ein und wurde nicht berücksichtigt.) Nach dem Bericht des „Site Committee 4 entschied das EC 1993, der GA Berlin als Ort des ICM 98 zu empfehlen. Diese Entscheidung wurde auch von der isrealischen Delegation in Luzern (Dvoretzky, Lindenstrauss, Lubotzky) akzeptiert. Die Abstimmung, die ursprünglich erst für den 1.8. vorgesehen war, fand schon am Schluf der Nachmittagssitzung am 31.7. ohne vorherige Diskussion statt und fiel einstimmig für Berlin aus. (In Kobe war dem Votum für Zürich noch eine lange Aussprache vorausgegangen.) Am nächsten Tag dankten Herr Grötschel (Vorsitzender der DMV) und Herr Hirzebruch (Vorsitzender des vorläufigen Organisationskomitees) der GA für das Vertrauen, stellten das Plakat für den Berliner ICM vor, verteilten eine Broschüre dazu und wiesen darauf hin, daf Informationen be reits per Computer auf dem ${ }_{n}$ World Wide Web" bei ${ }^{\text {Mosaic }}$ unter ,http://icm98.zib-berlin.de ${ }^{4}$ abgerufen werden können. Dann kündigte Yang (China) eine
Bewerbung für Peking 2002 an, was von Sloan sofort mit einer Gegenbewerbung von Australien gekontert wurde.

Über die meisten TOPs der GA ist schnell berichtet. Die Wahlen waren gut vorbereitet worden, wobei die Vorschläge des EC-nach fachlichen und geographischen Gesichtspunkten ausgewogen-nicht die Möglichkeit einer Auswahl zwischen verschiedenen Kandidaten vorsahen. Das neue EC der IMU sieht 1995-98 wie folgt aus: D. Mumford (USA, Prăsident), V. Arnold (Rubland), A. Dold (D, Vizeprăsident), J. Palis (Brasilien, Sekretăr), J. Arthur (Kanada), S. Donaldson (England), B. Enquist (Schweden), S.Mori (Japan), K. Parthasarathy (Indien) sowie J.L. Lions (Frankreich, Ex-Präsident). (Wie erwartet konnten sich zwei von der GA kurzfristig nominierte Gegenkandidaten nicht durchsetzen). Die Listen des EC für die "Commission on Development and Exchange (CDE) ${ }^{\mu}$ (Vorsitzender nach M.S. Narashiman ab 1995 R.Rebolledo, Chile) und das Exekutivkomitee der "International Commission on Mathematical Education (ICMI) (Vorsitzender bleibt $M$. de Guzman, Spanien) wurden abgehakt. Folgende Lander sind neue Mitglieder der IMU: Armenien, Kasachstan, Tunesien. Chile und der Iran steigen von Gruppe I in Gruppe II auf. Israel, das viele Mathematiker und -innen aus der früheren Sowjetunion aufgenommen hat, wird nach Gruppe IV hochgestuft. 
Lăngere Diskussionen gab es zum Thema „World Mathematical Year (WMY) $2000^{\mu}$ und "Format of future ICMs ${ }^{u}$. Der Präsident der IMU hatte am 6. Mai 1992 in Rio de Janeiro 2000 zum WMY erklärt; es wird von der UNESCO unterstützt. In einer weit verbreiteten Erklärung waren damals einige Punkte angesprochen worden, welche sich in einer von der GA jetzt verabschiedeten Resolution widerspiegeln: "The General Assembly ... asks the new EC to proceed with the planning of WMY 2000, and to organize and coordinate activities such as (a) an invitation to a select group of outstanding mathematicians to present their views on topics they expect to be central to mathematical activity in the next century, (b) the selection of a number of symposia, some possibly organized together with other scientific bodies, dedicated to mathematics, to its applications, and to its role in society, (c) events held under the auspecies of ICMI, CDE, ...". Für ein neues, ergänzendes Format Internationaler Mathematischer Kongresse setzte sich der zukünftige Präsident der IMU ein. Angenommen wurde dazu folgende Entschließung: "The GA recommends that the Programme Committee (i) explore ways of involving more effectively mathematicians from applied areas in the next Congress, including the possibility of introducing sessions co-sponsored with other scientific bodies; (ii) schedule some less formal scientific events of broad interest during the Congress."

Nach einer weiteren Empfehlung soll in Zukrunft der Name des Vorsitzenden des Programmlsomitees direkt nach seiner Ernennung bekanntgegeben werden (und nicht erst beim betreffenden ICM). Die Delegation aus den USA schlug unter "Verschiedenes" eine Resolution vor, wonach das EC darauf hinarbeiten solle, "to increase the representation of qualified women and improve the balance of representation of all ethnic groups in the committees that plan ICM 98 as well as those who are actually invited to be speaker". Da fragte der Delegierte Kameruns (Hogbe-Nlend), was denn bitte „ethnic groups ${ }^{4}$ seien. Ein russischer Delegierter (ab 1995 Vizepräsident. der IMU) merkte sarkastisch an: "You forgot the sexual minorities." Als dann Frau Sos, Delgierte Ungarns, erklärte, "this resolution in itself contains a discrimination", lehnte die GA eine Abstimmung. ab.-Die náchste Generalversammlung der IMU wird 1998 in Dresden oder Potsdam abgehalten.

\section{Council Meeting der EMS in Zürich}

\section{von Ina Kersten}

Die European Mathematical Society (EMS), die im Oktober 1990 in Warschau gegrindet wurde, be steht zur Zeit aus ungefăhr $\mathbf{4 5}$ korporativen Mitgliedern, wozu auch die DMV gehört, und 1700 persönlichen Mitgliedern. Die EMS wird von einem Executive Committee geleitet, das sich zwei- bis dreimal jährlich an wechselnden Orten in Europa trifft und ab 1995 aus folgenden Mitgliedern bestehen wird: Präsjdent: J.-P. Bourguignon (Frankreich); Vizepräsidenten: L. Màrki (Ungarn) und D.A.R. Wallace (Großbritanien); Schriftführer: P.W. Michor (Österreich); Schatzmeister: A. Lahtinen (Finnland); weitere Mitglieder: E. Bayer-Fluckiger (Frankreich), A. Conte (Italien); I.S. Labouriau (Portugal), A. Pelczar (Polen) und V.A. Solonnikov (RuBland). Alle zwei Jahre, und zwar wechselweise in Verbindung mit dem ECM (European Congress of Mathematics) und dem ICM, tagt der Council der EMS, der aus Delegierten der Mitglieder besteht. Zuletzt tagte der Council am 12. und 13. August 1994 in Zürich im AnschluB an den ICM. Es nahmen über 50 Delegierte teil, und dabei waren aus Deutschland die drei DMV-Delegierten K.-D. Bierstedt, F. Hirzebruch und I. Kersten, der GAMM-Präsident R. Mennicken und W. Schwarz als einer der Delegierten der persönlichen EMSMitglieder. Zu den Aufgaben des Councils gehört es beispielsweise, neue korporative EMS-Mitglieder und die Mitglieder des Excecutive Committee der EMS zu wählen. So wurde in Zürich auch ein neuer Präsident gewāhlt, da die Amtszeit des ersten und noch amtierenden EMS-Präsidenten $F$. Hirzebruch aus Bonn zum Jahresende 1994 auslăuft. Weiter wurden in Zürich Berichte über die zum Teil sehr erfolgreiche Arbeit der folgenden EMS-Kommissionen gegeben und darüber diskutiert: Verbindungen zur EG, Anwendingen der Mathematik, Frauen und Mathematik, Entwicklungsländer, Mathematische Aus bildung, Reviewing Journals wie Math. Reviews und Zentralblatt, Eurokonferenzen und Sommerschulen, Publikationen (hier wurde die Frage, ob und in welcher Form die EMS eine eigene Zeitschrift herausgeben soll, sehr kontrovers diskutiert und konnte noch nicht entschieden werden), Unterstitzung der osteuropăischen Mathematiker und Mathematikerinnen (hierfür hatte das Executive Committee 10000 ECU zur Verfügung gestellt und dies im Dezember 1993 in den EMS-Newsletter belainntgegeben; aber es losmen dann nur sehr wenige Bewerbungen, vermutlich, weil die Newsletterzu wenig gelesen wurden). Die Berichte der Kommissionen sind zum Teil schon in den EMS-Newsletter veroffentlicht worden oder werden dort noch erscheinen. Das Execntive Committee hat 